\title{
Selection of variables for stabilizing control using pole vectors
}

\author{
Kjetil Havre* \\ Sigurd Skogestad ${ }^{\dagger}$ \\ Department of Chemical Engineering \\ Norwegian University of Science and Technology (NTNU) \\ N-7491 Trondheim, Norway. \\ Accepted for publication in IEEE Trans. Autom. Control \\ Special issue on New developments and applications in performance limitations of feedback control
}

This version: May 15, 2003

\begin{abstract}
For a linear multivariable plant, it is known from earlier work that the easy computable pole vectors provide useful information about in which input channel (actuator) a given mode is controllable and in which output channel (sensor) it is observable. In this paper we provide a rigorous theoretical basis for the use of pole vectors, by providing a link to previous results on performance limitations for unstable plants.
\end{abstract}

\section{Introduction}

Most available control theories consider the problem of designing an optimal multivariable controller for a well-defined case with given inputs, outputs, measurements, performance specifications, and so on. The following important structural decisions (e.g. Skogestad and Postlethwaite (1996)) that come before the actual controller design are therefore not considered:

1. Selection of inputs $u$ (manipulated variables, actuators)

2. Selection of primary outputs $y_{1}$ : controlled variables with specified reference values

3. Selection of secondary outputs (measurements, sensors) $y_{2}$ : Extra variables that we select to measure and control in order to stabilize the plant and achieve local disturbance rejection.

4. Selection of control configuration: Structure of the subcontrollers that interconnect the above variables.

*Presently at Scandpower, Kjeller, Norway. Email: Kjetil. Havre@ scandpower. com
†Correspondence author. Email: skoge@ chemeng. nt nu. no, Phone: (+47) 73594154 


\section{Selection of controller type (control law specification, e.g. PID-control, LQG, etc.)}

Most industrial control systems are hierarchically structured with at least two layers. In the lower (secondary, regulatory) control layer, we have local control of the selected secondary controlled variables $y_{2}$. The controllers at this level are in most cases single-input-single-output (SISO) controllers. The reference values $\left(r_{2}\right)$ for these secondary variables are degrees of freedom (inputs) for the upper (primary, master, supervisory) control layer which deals with the control of the primary outputs $y_{1}$. The primary control layer may use multivariable or decentralized controller. The relative gain array (RGA) (Bristol, 1966) is a simple and popular tool for evaluating whether to use multivariable control, and to assist in the possible selection of input-output pairings for decentralized control. Specifically, pairing on negative steady-state RGA-elements should be avoided, because otherwise the sign of the steady-state gain will change if a loop is somehow taken out of service, which leads to instability if the loop contains integral action. However, this paper deals with the input-output pairing problem for the secondary control layer, with focus on stabilizing control. Here the RGA is not usually a very useful tool, because (i) interactions in this layer are usually small, (ii) stabilizing loops are not taken out of service, and (iii) output performance is not an important issue in this layer.

The objective of this paper is to find a simple tool for selecting inputs $u$ (actuators) and outputs $y$ (sensors) for stabilizing control, which is a subproblem of decisions 1 and 3 as listed above. Intutively, the classical concepts of state controllability and observability seem useful, since we want to select inputs such that the unstable states are easily controlled (excited), and select outputs such that the unstable states are easily observed. This leads one to consider the easily computable input pole vectors (directions) $\boldsymbol{u}_{p}$ and output pole vectors $\boldsymbol{y}_{p}$ as a tool for selecting inputs and outputs for stabilizing control. This approach also makes it possible to consider the inputs (state controllability) and outputs (observability) separately. Such ideas have been around in the literature since the 1960's, and, although we could not find it clearly stated, it has surely been used by practicioners. The basis for our work, came an attempt to design a stabilizing control system for the Tennessee-Eastman challenge problem (Downs and Vogel, 1993), where we found that the pole vectors provided very useful information for selecting inputs and outputs. This led us to search for a more rigorous basis for the use of pole vectors, and we were able to derive a direct link between the pole vectors and the minimum norm of the transfer function $K S$ from plant outputs (noise, disturbances) to plant inputs, both in terms the $\mathcal{H}_{2^{-}}$and $\mathcal{H}_{\infty}$-norms. This is clearly relevant, since an important issue for stabilizing control is to find an input-output pairing such that the input usage is minimized. First, this reduces the likelihood for input saturation (which most likely will result in instability), and second, it minimizes the "disturbing" effect of the stabilization of the remaining control problem. More specifically, for a plant $y=G u+G_{d} d$ with feedback control $u=-K(y+n-r)$ the closed-loop input signal is

$$
u=-K S(\underbrace{n+G_{d} d}_{\text {unavoidable }}-r)
$$

where $S=(I+G K)^{-1}$. Thus, to minimize the required (unavoidable) input usage $(u)$ due to measurement noise $(n)$ and disturbances $(d)$, we should choose input-output pairings for stabilizing control such that we minimize the resulting magnitude of the stabilized transfer function $[K S]_{j k}$ from the selected output $y_{k}$ to the selected input $u_{j}$. Note that the transfer function $K S$ should also be minimized in order to maximize the robustnes with respect to additive uncertainty (e.g., Glover (1986)). However, the presence of an unstable (Right half plane - RHP) pole imposes limitations on the achievable control performance (Zames, 1981), (Glover, 1986), (Francis, 1987), (Doyle et al., 1992), (Havre and Skogestad, 2001), including a bound on the minimum norm of $K S$. 
In summary, the main contribution in this paper is to provide a rigorous link between the concept of pole vectors and previous work on control performance limitations. The presentation in this paper is brief in places, and for detailed proofs and additional examples we refer to Chapter 6 of the thesis by Havre (1998).

Notation is fairly standard. We consider a linear plant with state-space realization

$$
\frac{d x(t)}{d t}=A x(t)+B u(t), \quad y=C x(t)+D u(t)
$$

where $t$ is time, $x(t) \in \mathbb{R}^{n}$ is the state, $u(t) \in \mathbb{R}^{m}$ is the input, $y(t) \in \mathbb{R}^{l}$ is the output, and $A, B, C, D$ are real matrices of appropriate dimensions. The corresponding transfer function matrix from inputs $u$ to outputs

$$
G(s)=C(s I-A)^{-1} B+D \stackrel{s}{=}\left[\begin{array}{l|l}
A & B \\
\hline C & D
\end{array}\right]
$$

We will use the following indexes (subscripts): $i$ for the states $x, j$ for the inputs $u$, and $k$ for the outputs $y$. We let $p_{i}=\lambda_{i}(A)$ denote the $i$ 'th pole of $G(s)$, where $\lambda_{i}(A)$ is the $i$ 'th eigenvalue of $A$. When we refer to the "mode" $p_{i}$ we mean the dynamic response associated with $p_{i}$. The $\mathcal{H}_{\infty}$-norm of the system $M$ is

$$
\|M(s)\|_{\infty}=\sup _{\omega} \bar{\sigma} M(j \omega)
$$

and the $\mathcal{H}_{2}$-norm of $M$ is

$$
\|M(s)\|_{2}=\sqrt{\frac{1}{2 \pi} \int_{-\infty}^{\infty} \operatorname{tr}\left(M(j \omega)^{H} M(j \omega)\right) d \omega}
$$

\section{Pole vectors}

For a pole $p_{i}$ the corresponding right eigenvector $\boldsymbol{t}_{i}$ ("output state direction") and left eigenvector $\boldsymbol{q}_{i}$ ("input state direction") are defined by

$$
A \boldsymbol{t}_{i}=p_{i} \boldsymbol{t}_{i} ; \quad \boldsymbol{q}_{i}^{H} A=p_{i} \boldsymbol{q}_{i}^{H}
$$

We usually normalize the eigenvectors to have unit length, i.e. $\left\|\boldsymbol{t}_{i}\right\|_{2}=1$ and $\left\|\boldsymbol{q}_{i}\right\|_{2}=1$. The input pole vector associated with the pole $p_{i}$ is defined as

$$
\boldsymbol{u}_{p, i}=B^{H} \boldsymbol{q}_{i}
$$

and the output pole vector is defined as

$$
\boldsymbol{y}_{p, i}=C \boldsymbol{t}_{i}
$$

For a given realization $(A, B, C, D)$ and normalized eigenvectors, the pole vectors corresponding to a distinct pole $p_{i}$ are unique up to the multiplication of a complex scalar $c$ of length $1(|c|=1)$. For a repeated pole $p_{i}$ (not distinct) there may be more than one linearly independent eigenvector, in which case the eigenvectors and pole vectors associated with $p_{i}$ are matrices. (These technical issues are not important for this paper, since all theorems are for distinct poles.) To motivate the introduction of pole vectors, consider for the case when all $n$ poles are distinct the following dyadic expansion of the transfer function,

$$
G(s)=\sum_{i=1}^{n} \frac{1}{\boldsymbol{q}_{i}^{H} \boldsymbol{t}_{i}} \cdot \frac{C \boldsymbol{t}_{i} \boldsymbol{q}_{i}^{H} B}{s-\lambda_{i}}+D=\sum_{i=1}^{n} \frac{1}{\boldsymbol{q}_{i}^{H} \boldsymbol{t}_{i}} \cdot \frac{\boldsymbol{y}_{p, i} \boldsymbol{u}_{p, i}^{H}}{s-\lambda_{i}}+D
$$


Note here that $\boldsymbol{t}_{i} \boldsymbol{q}_{i}^{H}$ is a rank-one $n \times n$ matrix and $\boldsymbol{y}_{p, i} \boldsymbol{u}_{p, i}^{H}$ is a rank-one $l \times m$ matrix, whereas the inner product $\boldsymbol{q}_{i}^{H} \boldsymbol{t}_{i}$ is a scalar. Douglas and Athans (1996) note that $\boldsymbol{u}_{p, i}=B^{H} \boldsymbol{q}_{i}$ is "an indication of how much the $i$ 'th mode is excited by the inputs", and that $\boldsymbol{y}_{p, i}=C \boldsymbol{t}_{i}$ is "an indication of how much the $i$ 'th mode is observed in the outputs". Indeed, the pole vectors may be used for checking the state controllability and observability of a system, and from linear system theory we have that (Zhou et al., 1996, p.52)).

- The mode $p_{i}$ is controllable if and only if $\boldsymbol{u}_{p, i}=B^{H} \boldsymbol{q}_{i} \neq 0$ (for all left eigenvectors $\boldsymbol{q}_{i}$ associated with $p_{i}$ ).

- The mode $p_{i}$ is observable if and only if $\boldsymbol{y}_{p, i}=C \boldsymbol{t}_{i} \neq 0$ (for all right eigenvectors $\boldsymbol{t}_{i}$ associated with $\left.p_{i}\right)$.

It follows that a system is controllable (observable) if and only if every mode $p_{i}$ is controllable (observable). Furthermore, a mode $p_{i}$ is controllable from an input $u_{j}$ if the $j^{\prime}$ the element in $\boldsymbol{u}_{p, i}$ is nonzero, and observable from an output $y_{k}$ if the $k$ 'the element in $\boldsymbol{y}_{p, i}$ is nonzero.

From the latter results it seems clear that the magnitudes of elements in the input pole vector $\boldsymbol{u}_{p, i}$ give information about from which input the $i$ 'th mode is most controllable, and that the magnitude of the elements in the output pole vector $\boldsymbol{y}_{p, i}$ give information about in which output the $i$ 'th mode is most observable. The objective of this paper is to confirm this intuition in terms of which input and output to select for stabilizing control.

REMARK 1. The pole vectors are easy to compute as part of an eigenvalue computation, but one needs to be a bit careful to get the same order for the left and right eigenvectors. Matlab routines for their calculation are available from the home page of S. Skogestad.

REMARK 2. The inner product $\boldsymbol{q}_{i}^{H} \boldsymbol{t}_{i}$ of the eigenvectors influences the magnitude of the transfer function and thus the magnitude of the input usage, but does not influence the relative ranking of candidate inputs and outputs.

The following example illustrates how the pole vectors may be useful for practical applications.

EXAmPle 1 The Tennessee Eastman chemical process (Downs and Vogel, 1993) was introduced as a challenge problem to test methods for control structure design. The process has 12 manipulated inputs and 41 candidate measurements, of which we here consider 11. The open-loop process is unstable, and the first step in a control system design for this process is to design a stabilizing control system. To assist in this step we compute the pole vectors. The model has six unstable poles in the operating point considered

$$
p_{i}=\left[\begin{array}{llll}
0 & 0.001 & 0.023 \pm 0.156 j & 3.066 \pm 5.079 j
\end{array}\right]
$$

The inner products of the left and right eigenvectors corresponding to the unstable modes are

$$
q_{i}^{H} t_{i}=\left[\begin{array}{llll}
0.3209 & 0.0467 & 0.0210 & 0.0074
\end{array}\right]
$$

The output pole vectors are

$$
\left|Y_{p}\right|=\left[\begin{array}{llll}
0.000 & 0.001 & 0.041 & 0.112 \\
0.000 & 0.004 & 0.169 & 0.065 \\
0.000 & 0.000 & 0.013 & 0.366 \\
0.000 & 0.001 & 0.051 & 0.410 \\
0.009 & 0.580 & 0.488 & 0.315 \\
0.000 & 0.001 & 0.041 & 0.115 \\
1.605 & 1.192 & 0.754 & 0.131 \\
0.000 & 0.001 & 0.039 & 0.107 \\
0.000 & 0.001 & 0.038 & 0.217 \\
0.000 & 0.001 & 0.055 & 1.485 \\
0.000 & 0.002 & 0.132 & 0.272
\end{array}\right]
$$


where we have taken the absolute value to avoid complex numbers in the vectors, and we have combined eigenvector pairs corresponding to a complex eigenvalue into a single column. The first column corresponds to the pole $p_{1}=0$, the second column corresponds to the pole $p_{2}=0.001$, the third column corresponds to the complex conjugate pair $p_{3,4}=0.023 \pm 0.156 j$ and the fourth column corresponds to the complex conjugate pair $p_{5,6}=3.066 \pm 5.079 j$. From the output pole vectors, we see that the pole at $p_{1}=0$ is observable in output $7, p_{2}$ in outputs 5 and $7, p_{3,4}$ mostly in outputs 5 and 7, and $p_{5,6}$ mostly in output 10 . The input pole vectors are

$$
\left|U_{p}\right|=\left[\begin{array}{cccc}
6.815 & 6.909 & 2.573 & 0.964 \\
6.906 & 7.197 & 2.636 & 0.246 \\
0.148 & 1.485 & 0.768 & 0.044 \\
3.973 & 11.550 & 5.096 & 0.470 \\
0.012 & 0.369 & 0.519 & 0.356 \\
0.597 & 0.077 & 0.066 & 0.033 \\
0.132 & 1.850 & 1.682 & 0.110 \\
22.006 & 0.049 & 0.000 & 0.000 \\
0.007 & 0.054 & 0.009 & 0.013 \\
0.247 & 0.708 & 1.501 & 2.020 \\
0.109 & 0.976 & 1.446 & 0.753 \\
0.033 & 0.094 & 0.201 & 0.302
\end{array}\right]
$$

From the input pole vectors, we see that the pole at $p_{1}=0$ is most easily controllable from input $8, p_{2}$ from input 4 , $p_{3,4}$ from input 4 and $p_{5,6}$ from input 10.

When designing a stabilizing control system, we normally start by stabilizing the "most unstable" (fastest) mode with the largest absolute value, i.e. pole $p_{5,6}$ in this case. From the pole vectors, this mode is most easily stabilized by use of input 10 (reactor cooling water flow) to control output 10 (the reactor cooling water outlet temperature). We designed a simple PI-controller for this loop and recomputed the poles. In addition to stabilizing the mode corresponding to $p_{5,6}$, the recomputation of the system poles shows that the closing of this single loop also stabilizes the mode corresponding to $p_{3,4}$, which is reasonable since the the pole vectors show that this mode is observable in output 10 and controllable from input 10. The stabilization of the two remaining integrators $\left(p_{1}\right.$ and $\left.p_{2}\right)$ requires the closing of two additional loops (two liquid level loops). For more details see (Havre, 1998) and (Havre and Skogestad, 1998).

The above example demonstrates the practical usefulness of pole vectors. The objective of the remaining of this paper is to rigorously link the pole vectors to existing results on achievable performance.

\section{Stabilizing control with minimum input energy $\left(\mathcal{H}_{2}\right.$-norm)}

\subsection{SISO control}

A critical issue is usually to avoid saturation of the input used for stabilization, because otherwise the system effectively becomes open-loop and stability is lost. More generally, it is desirable to minimize the input usage required for stabilization, and this motivates the following problem:

PROBLEM 1 (SISO input energy for stabilization, see Figure 1). Consider a plant $G$ with a single mode $^{1}$ $p \in \mathbb{C}_{+}(\operatorname{Re} p>0)$ and white measurement noise $n_{k}$ of unit intensity in each output $y_{k}$. Find the best

\footnotetext{
${ }^{1}$ We consider a specific pole $p=p_{i}$ and the subscript $i$ is omitted in the following.
} 


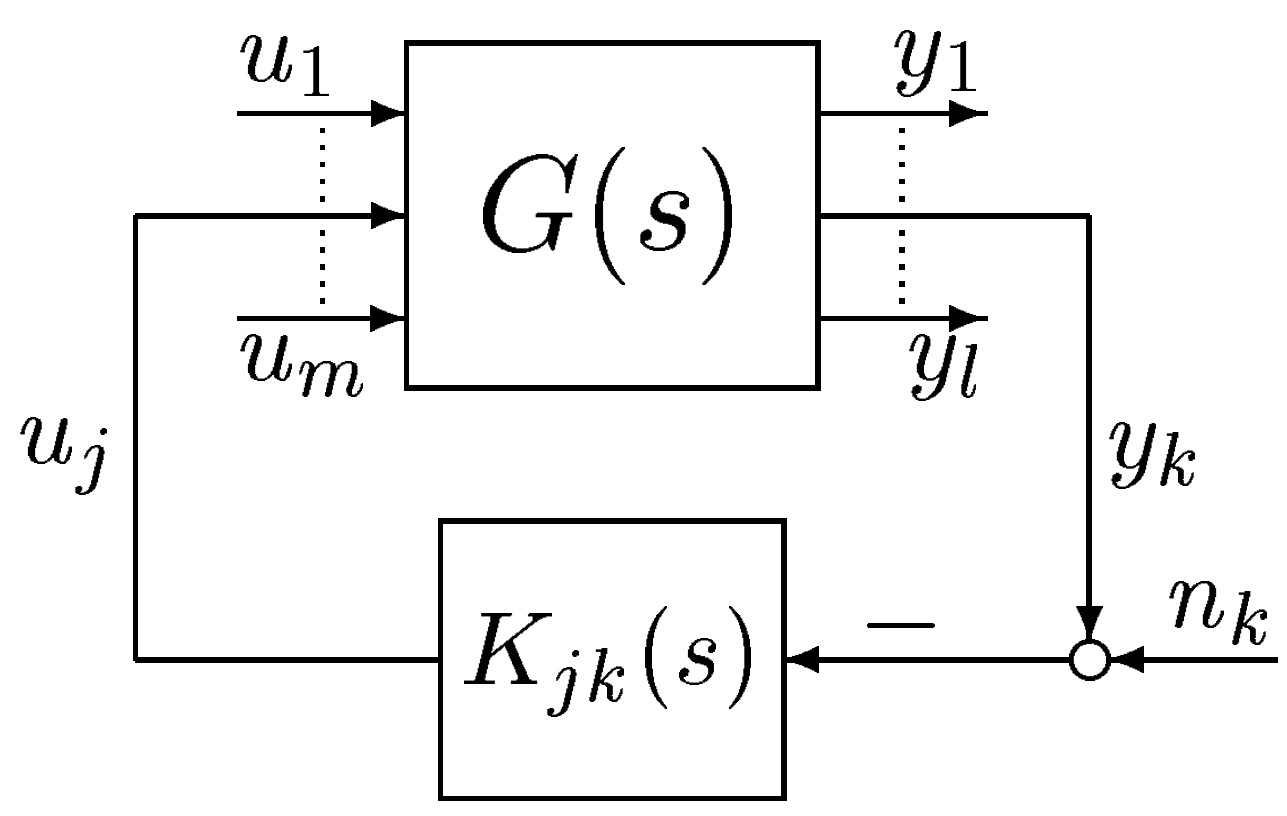

Figure 1: Plant $G$ with stabilizing control loop $u_{j} \leftrightarrow y_{k}$

pairing $u_{j} \leftrightarrow y_{k}$, such that the plant is stabilized with minimum expected input energy

$$
J(j, k)=E\left\{\lim _{T \rightarrow \infty} \frac{1}{T} \int_{0}^{T} u_{j}^{2}(t) d t\right\}
$$

At first sight it is not clear that the output selection problem is included at all, since the outputs do not enter into the objective (4) explicitly. However, the output selection problem is included implicitly through the measurement noise and the expectation operator $E$. This important problem has attracted little attention in the system theory literature, although there is some related work (Wang and Davison, 1973; Benninger, 1986; Tarokh, 1985; Tarokh, 1992; Lunze, 1992). For this problem an analytical solution can be found in terms of the pole vectors:

TheOrem 1 (Solution to Problem 1). The minimum input energy J, for a specific input $j$ and output $k$ is

$$
J(j, k)_{\min }=\frac{8 p^{3}\left(\boldsymbol{q}^{H} \boldsymbol{t}\right)^{2}}{u_{p, j}^{2} y_{p, k}^{2}}
$$

where $p$ is the pole, $u_{p, j}$ is the $j$ 'th element in the input pole vector, $y_{p, k}$ is the $k$ 'th element in the output pole vector, and $\boldsymbol{q}$ and $\boldsymbol{t}$ are the left and right eigenvectors corresponding to the mode $p$. Stabilization is impossible for the pair $(j, k)$, even with infinite input energy, if $u_{p, j}=0$ (the mode $p$ is not controllable from input $j$ ) or $y_{p, k}=0$ (the mode $p$ is not observable from output $k$ ). The numerator in (5) is independent of the selection of input and output. Hence, to minimize the input energy required for stabilization with SISO control one should

- Select the input $j$ corresponding to the largest entry $\left|u_{p, j}\right|$ in the input pole vector $\boldsymbol{u}_{p}$.

- Select the output $k$ corresponding to the largest entry $\left|y_{p, k}\right|$ in the output pole vector $\boldsymbol{y}_{p}$. 
Proof of (5). Because of the separation theorem we may prove (5) by first finding the best input using state feedback (LQR) under the assumption of perfect measurement of all states, and then constructing the optimal state observer (LQE).

LQR: Optimal state feedback to input $u_{j}$. In this case, the problem is to minimize the input usage due to non-zero initial states $x_{0}$, i.e. minimize the deterministic cost $J_{\mathrm{LQR}}(j)=\int_{0}^{\infty} u_{j}^{2}(t) d t$. The corresponding Riccati equation with zero weight on the states and unity weight on the input becomes $A^{T} X+X A-X B e_{j} e_{j}^{T} B^{T} X=0$, where $e_{j}$ is a unit vector with 1 in position $j$ and 0 in the other elements. With a single real pole $p$ the solution is $X=\frac{2 p}{u_{p, j}^{2}} \boldsymbol{q q}^{T} \geq 0$ and the optimal state feedback gain becomes

$$
K_{j}=e_{j}^{T} B^{T} X=\frac{2 p}{u_{p, j}} \boldsymbol{q}^{T}
$$

LQE: Kalman filter (state observer) based on $y_{k}$. There is no process noise and the Riccati equation becomes $Y A^{T}+A Y-Y C^{T} e_{i} e_{i}^{T} C Y=0$. The solution is $Y=\frac{2 p}{y_{p, k}^{2}} \boldsymbol{t} \boldsymbol{t}^{T} \geq 0$ so the optimal feedback gain from output $y_{k}$ to the state estimate becomes

$$
K_{f, k}=Y C^{T} e_{k}=\frac{2 p}{y_{p, k}} \boldsymbol{t}
$$

Finally, to obtain the value of the expected input energy $J$, we use (Kwakernaak and Sivan, 1972, Theorem 5.4 part (d) page 394-395).

$$
J(j, k)=\operatorname{tr}\left\{X K_{f, k} K_{f, k}^{T}\right\}=\operatorname{tr}\left\{\frac{2 p}{u_{p, j}^{2}} \boldsymbol{q q}^{T} \frac{2 p}{y_{p, k}} \boldsymbol{t} \frac{2 p}{y_{p, k}} \boldsymbol{t}^{T}\right\}=\frac{8 p^{3}}{u_{p, j}^{2} y_{p, k}^{2}}\left(\boldsymbol{q}^{T} \boldsymbol{t}\right)^{2}
$$

\subsection{MIMO control}

We here consider the same problem as above, but with multivariable (MIMO) control.

THEOREM 2 (MIMO input energy for stabilization). Consider a plant $G$ with a single unstable mode $p \in \mathbb{C}_{+}$and with white measurement noise $n_{k}$ of unit intensity in each output $y_{k}$. The minimal achievable input energy required for stabilization,

$$
J=E\left\{\lim _{T \rightarrow \infty} \frac{1}{T} \int_{0}^{T} u^{T}(t) u(t) d t\right\}
$$

is given in terms of the pole vectors:

$$
J_{\text {min }}=\frac{8 p^{3} \cdot\left(\boldsymbol{q}^{T} \boldsymbol{t}\right)^{2}}{\left\|\boldsymbol{u}_{p}\right\|_{2}^{2} \cdot\left\|\boldsymbol{y}_{p}\right\|_{2}^{2}}
$$

By comparing the minimum value of $J(j, k)$ (SISO control) with the minimum value of $J$ (MIMO control), we can quantify the extra input energy needed to stabilize the plant using SISO control compared to full multivariable control. As expected, this is directly given by the relative magnitudes of the elements in the pole vectors:

$$
\frac{\sqrt{J(j, k)_{\min }}}{\sqrt{J_{\min }}}=\frac{\left\|\boldsymbol{u}_{p}\right\|_{2} \cdot\left\|\boldsymbol{y}_{p}\right\|_{2}}{\left|u_{p, j}\right| \cdot\left|y_{p, k}\right|} \geq 1
$$




\subsection{Interpretation in terms of the $\mathcal{H}_{2}$-norm}

The above theorems may alternatively be interpreted in terms of the $\mathcal{H}_{2}$-norm of the closed-loop transfer function $K S$ from plant inputs to plant outputs. This follows since (e.g. (Zhou et al., 1996)):

$$
\begin{gathered}
\min _{K_{j k}}\left\|K_{j k} S_{k k}(s)\right\|_{2}=\sqrt{J(j, k)_{\min }} \quad \text { where } \quad S_{k k}(s)=\left(1+G_{k j} K_{j k}(s)\right)^{-1} \\
\min _{K}\|K S(s)\|_{2}=\sqrt{J_{\min }} \quad \text { where } \quad S(s)=(I+G K)^{-1}
\end{gathered}
$$

\section{Stabilizing control with minimum input usage $\left(\mathcal{H}_{\infty}\right.$-norm)}

Interestingly, almost identical results can be derived in terms of the $\mathcal{H}_{\infty}$-norm. Thus, the $\mathcal{H}_{2^{-}}$and $\mathcal{H}_{\infty}$ norms give the same best input-output pairing for stabilizing a plant $G$ with a single unstable mode.

THEOREM 3 (Stabilizing SISO Control with minimum $\mathcal{H}_{2}$ and $\mathcal{H}_{\infty}$ input usage). Consider a plant $G$ with a single unstable mode $p \in \mathbb{C}_{+}$. The minimum achievable $\mathcal{H}_{2}$ - and $\mathcal{H}_{\infty}$-norm of the closed-loop transfer function $K_{j k} S_{k k}$ from output $y_{k}$ to the input $u_{j}$ is then

$$
\min _{K_{j k}(s)}\left\|K_{j k} S_{k k}(s)\right\|_{\infty}=\frac{1}{\sqrt{|2 p|}} \min _{K_{j k}(s)}\left\|K_{j k} S_{k k}(s)\right\|_{2}=\left|\left(G_{k j}\right)_{s}^{-1}(p)\right|=\frac{|2 p| \cdot\left|\boldsymbol{q}^{H} \boldsymbol{t}\right|}{\left|u_{p, j}\right| \cdot\left|y_{p, k}\right|}
$$

where $u_{p, j}$ is the $j$ 'th element in the input pole vector, $y_{p, k}$ is the $k$ 'th element in the output pole vector, $\boldsymbol{q}$ and $\boldsymbol{t}$ are the left and right eigenvectors of $A$ corresponding to the pole $p, S_{k k}(s)=\left(1+G_{k j} K_{j k}(s)\right)^{-1}$, and the notation $\left(G_{k j}\right)_{s}^{-1}(p)$ means: Find the stable version of $G_{k j}$ with the RHP-pole at $s=p$ mirrored across the imaginary axis, i.e., $\left(G_{k j}(s)\right)_{s}=\frac{s-p}{s+p} G_{k j}(s)$, take its inverse, i.e. $\left(G_{k j}(s)\right)_{s}^{-1}=\left(\left(G_{k j}(s)\right)_{s}\right)^{-1}$, and evaluate $\left(G_{k j}(s)\right)_{s}^{-1}$ at $s=p$.

REMARK 1. When minimizing the input usage, both in terms of the $\mathcal{H}_{2}$ - and $\mathcal{H}_{\infty}$-norms, the unstable open-loop pole $p$ is mirrored into the left half plane for the closed-loop system.

REMARK 2. The $\mathcal{H}_{\infty}$-controller that achieves the bound in (13) is in general improper.

Proof of Theorem 3. The identity $\min _{K_{j k}(s)}\left\|K_{j k} S_{k k}(s)\right\|_{\infty}=\left|\left(G_{k j}\right)_{s}^{-1}(p)\right|$ follows from Havre and Skogestad (2001, Theorem 4 and eq.(26)). Similar and more general problems have been considered in Francis (1987, Section 5.1). The last identity is proved as follows: Since $p$ is the only unstable mode, it follows from (3) that a partial fraction expansion of $G$ contains the following two terms

$$
G(s)=\frac{1}{\boldsymbol{q}^{H} \boldsymbol{t}} \cdot \frac{\boldsymbol{y}_{p} \boldsymbol{u}_{p}^{H}}{s-p}+N(s)
$$

where $N(s)$ is stable. Also, $\left(G_{k j}(s)\right)_{s}=e_{k}^{T} \frac{s-p}{s+p} G(s) e_{j}$ and since $y_{p, k}=e_{k}^{T} \boldsymbol{y}_{p}$ and $u_{p, j}=\boldsymbol{u}_{p}^{H} e_{j}$ we have

$$
\left|\left(G_{k j}\right)_{s}(p)=\right| \frac{1}{\boldsymbol{q}^{H} \boldsymbol{t}} \frac{y_{p, k} u_{p, j}}{s+p}+\left.\frac{s-p}{s+p} N_{k j}(s)\right|_{s=p}=\frac{\left|y_{p, k}\right| \cdot\left|u_{p, j}\right|}{|2 p| \cdot\left|\boldsymbol{q}^{H} \boldsymbol{t}\right|}
$$

The relationship to the $\mathcal{H}_{2}$-norm follows from Theorem 1 and (11). 
Theorem 3 has the following implication for actuator/measurement selection for a plant with a single unstable mode:

The required input usage for stabilization, both in terms of the $\mathcal{H}_{2}$ - and $\mathcal{H}_{\infty}$-norms, is minimized by selecting the output (measurement) $y_{k}$ corresponding to the largest element in the output pole vector $\boldsymbol{y}_{p}$, and the input (actuator) $u_{j}$ corresponding to the largest element in the input pole vector $\boldsymbol{u}_{p}$.

More precisely, we propose the following procedure for designing a SISO stabilizing controller, assuming that input usage is a concern:

1. Scale the plant inputs and outputs such that a unit change in each input $u_{j}$ is of equal importance, and a unit change in each output $y_{k}$ is of equal importance. Specifically, we have

$$
G=D_{y}^{-1} \hat{G} D_{u}
$$

where $\hat{G}$ denotes the original (unscaled) model, and the diagonal scaling matrices are

$$
D_{y}=\operatorname{diag}\left\{\hat{y}_{k, \max }\right\}, \quad D_{u}=\operatorname{diag}\left\{\hat{u}_{j, \max }\right\}
$$

Typically, $\hat{u}_{j, \max }$ denotes the maximum allowed input deviation, for example, the distance from the nominal input value to its saturation limit. Typically, $\hat{y}_{k, \max }$ denotes the magnitude of the measurement noise $(n)$ plus the expected output deviation due to disturbances (process noise) $\left(G_{d} d\right)$.

2. Compute the pole vectors $\boldsymbol{u}_{p}$ and $\boldsymbol{y}_{p}$.

3. Select an input $u_{j}$ corresponding to a large element in the input pole vector $\boldsymbol{u}_{p}$.

4. Select an output $y_{k}$ corresponding to a large element in the output pole vector $\boldsymbol{y}_{p}$.

5. Design a controller for this input/output pairing.

Obviously, the input magnitude is not the only concern when it comes to selecting an input/outputpairing for stabilizing control, and this is the reason for using the term "large" rather than "largest" in steps 3 and 4 .

EXAMPLE 2 Stabilization of chemical reactor. The objective is to design a stabilizing SISO controller for the exotermic continuously stirred tank reactor (CSTR) in Figure 2 using a SISO controller. The candidate actuators (inputs) are the outflow and inlet temperature, $u=\left[\begin{array}{c}F \\ T_{i n}\end{array}\right]$, and the candidate measurements (outputs) are the reactor volume (level) and the reactor temperature, $y=\left[\begin{array}{c}V \\ T\end{array}\right]$. The appropriately scaled linear model is

$$
G(s)=\left[\begin{array}{cc}
\frac{-20}{s} & 0 \\
\frac{-70}{s(s-3.5)} & \frac{20}{s-3.5}
\end{array}\right] \stackrel{s}{=}\left[\begin{array}{cc|cc}
0 & 0 & -1 & 0 \\
70 & 3.5 & 0 & 20 \\
\hline 20 & 0 & 0 & 0 \\
0 & 1 & 0 & 0
\end{array}\right]
$$




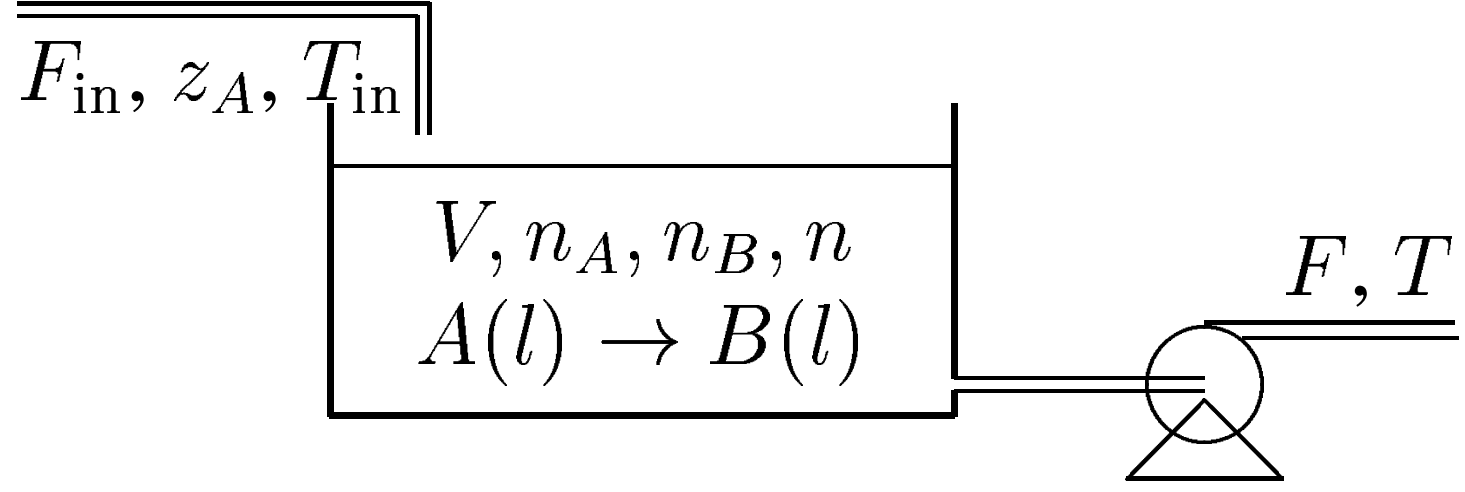

Figure 2: Chemical reactor (CSTR)

The pole at the origin $\left(p_{1}=0\right)$ is due to the integrating level, and the unstable pole at $p_{2}=3.5$ is due to the exothermic reaction. The corresponding pole vectors are

$$
\begin{gathered}
Y_{p}=\left[\begin{array}{ll}
\boldsymbol{y}_{p, 1} & \boldsymbol{y}_{p, 2}
\end{array}\right]=\left[\begin{array}{cc}
0.9988 & 0 \\
0.9988 & 1
\end{array}\right] \\
U_{p}=\left[\begin{array}{ll}
\boldsymbol{u}_{p, 1} & \boldsymbol{u}_{p, 2}
\end{array}\right]=\left[\begin{array}{cc}
-1 & -0.9988 \\
0 & 0.9988
\end{array}\right]
\end{gathered}
$$

and the inner products of the corresponding eigenvectors are $\boldsymbol{q}_{1}^{H} \boldsymbol{t}_{1}=0.05$ and $\boldsymbol{q}_{2}^{H} \boldsymbol{t}_{2}=0.05$. From $\boldsymbol{y}_{p, 2}$ we see that the unstable mode at $p_{2}=3.5$ is only observable in output 2 (this is also seen easily from $G(s)$ ), and from $\boldsymbol{u}_{p, 2}$ we see that the unstable mode is equally controllable in both inputs. Thus, to minimize the input usage required for stabilization we should use output 2 and any of the two inputs.

Comment: We note from $\boldsymbol{u}_{p, 1}$ that the pole at the origin $\left(p_{1}=0\right)$ is only controllable from input 1 , but observable in both outputs. This suggest that we may be able to move both the poles into the LHP if we design a controller using input 1 and output 2. This is indeed confirmed, for example, by designing a LQG-controller for the element $g_{21}(s)$.

REMARK. For this simple example, we reach the same conclusion easily by looking at the elements of $G(s)$, and indeed, an evaluation of the poles and zeros of the transfer function elements yields valuable insight. However, for more complicated cases the use of pole vectors avoids the combinatorial complexity of considering input/output-pairs and is also more reliable numerically.

\section{Discussion}

\subsection{Stable poles: Pole placement with minimum feedback gains}

The pole vector results in this paper, in terms of minimum input usage, apply only to an unstable (RHP) pole, because for a stable plant the minimum input usage is zero. However, from (6) and (7) we note that an alternative interpretation is that pairing on large elements in the pole vectors minimizes the required state feedback gain $K_{j}$ and observer gain $K_{f, k}$, and this result also generalizes to moving a stable (LHP) pole.

State feedback to input $u_{j}$. We want to move the distinct real open-loop pole $p$ to the closed-loop location $\mu$ by the use of state feedback from input $u_{j}$. The required state feedback gain vector is

$$
K_{j}=\frac{p-\mu}{u_{p, j}} \boldsymbol{q}^{T}
$$


where $u_{p, j}$ is the $j$ 'th element in the input pole vector corresponding to the pole $p$ and $\boldsymbol{q}$ is the corresponding left eigenvector. Here only the scalar $u_{p, j}$ depends on the choice of input $j$, so it follows that any matrix norm of $K_{j}$ is minimized by selecting the input $j$ corresponding to the largest element magnitude in the input pole vector $\boldsymbol{u}_{p}$.

State observer based on $y_{k}$. Similarly, we want to move the observer pole $p$ to the desired location $\nu$ by feedback from output $y_{k}$. The required observer feedback gain vector is

$$
K_{f, k}=\frac{p-\nu}{y_{p, k}} \boldsymbol{t}
$$

where $y_{p, k}$ is the $k$ 'th element in the output pole vector corresponding to the pole $p$ and $\boldsymbol{t}$ is the corresponding right eigenvector. Thus, the norm of $K_{f, k}$ is minimized by selecting the output $k$ corresponding to the largest element magnitude in the output pole vector $\boldsymbol{y}_{p}$.

The above results provide some theoretical basis for using the pole vectors as a tool selecting an input/output pair for moving a stable pole, including a pole located at the origin.

\subsection{Multiple unstable poles}

The main limitation with the theoretical results presented in this paper is that they only apply for cases with a single RHP-pole. For cases with multiple RHP-poles, the pole vectors associated with a specific RHP-pole give the input usage required to move this RHP-pole assuming that the other RHP-poles are unchanged. This is of course unrealistic and may lead to misleading results for some plants which are difficult to stabilize, for example, if we have a complex pair of RHP-poles with a RHP-zero nearby (Havre, 1998, Example 6.5).

Nevertheless, the pole vectors have proven themselves useful in several applications with more than one unstable mode, including the stabilizing control of the Teneessee-Eastman process (Havre, 1998) (Havre and Skogestad, 1998) with 6 unstable modes, and the selection of pressure sensor location for stabilization of desired two-phase flow regimes in pipelines (Havre et al., 2000) (Storkaas et al., 2001) which has a pair of complex RHP-poles. For such applications the pole vectors need to be interpreted with care and the results need to be checked, for example, by designing controllers. It is recommended to start by using the pole vectors of $G(s)$ to design a controller for the most unstable mode (furthest into the right half plane). Next, obtain the transfer function for the "new" partially stabilized plant, and repeat steps 2-5 until the plant is completely stabilized. In some cases, as illustrated in the reactor example, closing a single loop can stabilize more than one unstable mode.

\section{Conclusion}

The input and output pole vectors for a pole $p$ are defined as $\boldsymbol{u}_{p}=B^{H} \boldsymbol{q}$ (where $\boldsymbol{q}$ is the left eigenvector of $A$ corresponding to the pole $p$ ) and $\boldsymbol{y}_{p}=C \boldsymbol{t}$ (where $\boldsymbol{t}$ is the right eigenvector). The main contribution in this paper is to show that the pole vectors provide a simple and powerful tool for selecting inputs (actuators) and outputs (sensors) for stabilizing control, for cases where input usage is an important concern. More precisely, we show that the element magnitudes of the pole vectors are inversely related to the minimum input usage needed to stabilize one unstable mode using a SISO controller. This holds both in terms of minimum input energy with white noise and for the $\mathcal{H}_{2}$ - and $\mathcal{H}_{\infty}$-norms of the closed-loop transfer function 
$K S$ from plant outputs to plant inputs as given in Theorem 3:

$$
\min _{K_{j k}(s)}\left\|K_{j k} S_{k k}(s)\right\|_{\infty}=\frac{1}{\sqrt{|2 p|}} \min _{K_{j k}(s)}\left\|K_{j k} S_{k k}(s)\right\|_{2}=\left|\left(G_{k j}\right)_{s}^{-1}(p)\right|=\frac{|2 p| \cdot\left|\boldsymbol{q}^{H} \boldsymbol{t}\right|}{\left|u_{p, j}\right| \cdot\left|y_{p, k}\right|}
$$

where $u_{p, j}$ is the $j$ 'th element in the input pole vector, and $y_{p, k}$ is the $k$ 'th element in the output pole vector. Input usage is thus minimized by selecting an actuator (input) with a corresponding large value of $\left|u_{p, j}\right|$ and a sensor (output) with a corresponding large value of $\left|y_{p, k}\right|$. Furthermore, if one element in the pole vector dominates, see (10), there is little loss imposed by selecting only one actuator or one sensor.

\section{References}

Benninger, N. F. (1986). Proper choice of input and output variables by means of new consistent structure measures. Large Scale Systems:Theory and Applications 1, 161-166.

Bristol, E. H. (1966). On a new measure of interactions for multivariable process control. IEEE Transactions on Automatic Control AC-11, 133-134.

Douglas, J. and M. Athans (1996). Multivariable poles, zeros and pole-zero cancellations. In: The Control Handbook (W.S. Levine, Ed.). CRC Press \& IEEE Press. pp. 445-450.

Downs, J. J. and E. F. Vogel (1993). A plant-wide industrial process control problem. Computers Chem. Engng. 17(3), 245-255.

Doyle, J. C., B. Francis and A. Tannenbaum (1992). Feedback Control Theory. Macmillan Publishing Company.

Francis, B. (1987). A course in $\mathcal{H}_{\infty}$ control theory. Lecture Notes in Control and Information Sciences. Springer-Verlag. Berlin.

Glover, K. (1986). Robust stabilization of linear multivariable systems: relations to approximation. Int. J. Control 43, 741-766.

Havre, K. (1998). Studies on Controllability Analysis and Control Structure Design. PhD thesis. Norwegian University of Science and Technology, Trondheim. Available from homepage of S. Skogestad.

Havre, K. and S. Skogestad (1998). Selection of variables for regulatory control using pole vectors. In: Proc.IFAC symposium DYCOPS-5. Corfu, Greece. pp. 614-619.

Havre, K. and S. Skogestad (2001). Achievable performance of multivariable systems with unstable zeros and poles. International Journal of Control 74, 1131-1139.

Havre, K., K.O. Stornes and H. Stray (2000). Taming slug flow in pipelines. ABB Review (4), 55-63.

Kwakernaak, H. and R. Sivan (1972). Linear Optimal Control Systems. Wiley Interscience. New York.

Lunze, J. (1992). Feedback Control of Large-Scale Systems. Prentice-Hall. Englewood Cliffs. 
Skogestad, S. and I. Postlethwaite (1996). Multivariable Feedback Control, Analysis and Design. John Wiley \& Sons. Chichester.

Storkaas, E., S. Skogestad and V. Alstad (2001). Stabilization of desired flow regimes in pipelines. AIChE Annual meeting, Chicago 10-14 Nov., 1996, Paper 45f.

Tarokh, M. (1985). Fixed modes in multivariable systems using constrained controllers. Automatica 21(4), 495-497.

Tarokh, M. (1992). Measures for controllability, observability, and fixed modes. IEEE Transactions on Automatic Control 37(8), 1268-1273.

Wang, S. H. and E. J. Davison (1973). On the stabilization of decentralized control systems. IEEE Transactions on Automatic Control 18(5), 473-478.

Zames, G. (1981). Feedback and optimal sensitivity: model reference transformations, multiplicative seminorms, and approximate inverses. IEEE Transactions on Automatic Control AC-26(2), 301-320.

Zhou, K., J. C. Doyle and K. Glover (1996). Robust and Optimal Control. Prentice-Hall. Upper Saddle River. 